\title{
A Permanent-Magnet Microwave Ion Source for a Compact High-Yield Neutron Generator
}

\author{
O. Waldmann ${ }^{1}$, B. Ludewigt ${ }^{1}$ \\ ${ }^{1}$ Lawrence Berkeley National Laboratory \\ One Cyclotron Road \\ Berkeley, CA 94720
}




\section{DISCLAIMER}

This document was prepared as an account of work sponsored by the United States Government. While this document is believed to contain correct information, neither the United States Government nor any agency thereof, nor the Regents of the University of California, nor any of their employees, makes any warranty, express or implied, or assumes any legal responsibility for the accuracy, completeness, or usefulness of any information, apparatus, product, or process disclosed, or represents that its use would not infringe privately owned rights. Reference herein to any specific commercial product, process, or service by its trade name, trademark, manufacturer, or otherwise, does not necessarily constitute or imply its endorsement, recommendation, or favoring by the United States Government or any agency thereof, or the Regents of the University of California. The views and opinions of authors expressed herein do not necessarily state or reflect those of the United States Government or any agency thereof or the Regents of the University of California. 


\title{
A Permanent-Magnet Microwave Ion Source for a Compact High-Yield Neutron Generator*
}

\author{
O. Waldmann ${ }^{a}$ and B. Ludewigt ${ }^{a}$ \\ ${ }^{a}$ Lawrence Berkeley National Laboratory \\ 1 Cyclotron Road, Berkeley, CA 94720
}

\begin{abstract}
We present recent work on the development of a microwave ion source that will be used in a high-yield compact neutron generator for active interrogation applications. The sealed tube generator will be capable of producing high neutron yields, $5 \cdot 10^{11} \mathrm{n} / \mathrm{s}$ for $\mathrm{D}-\mathrm{T}$ and $\sim 1 \cdot 10^{10} \mathrm{n} / \mathrm{s}$ for D$\mathrm{D}$ reactions, while remaining transportable. We constructed a microwave ion source $(2.45 \mathrm{GHz})$ with permanent magnets to provide the magnetic field strength of $87.5 \mathrm{mT}$ necessary for satisfying the electron cyclotron resonance (ECR) condition. Microwave ion sources can produce high extracted beam currents at the low gas pressures required for sealed tube operation and at lower power levels than previously used RF-driven ion sources. A $100 \mathrm{~mA}$ deuterium/tritium beam will be extracted through a large slit $\left(60 \cdot 6 \mathrm{~mm}^{2}\right)$ to spread the beam power over a larger target area. This paper describes the design of the permanent-magnet microwave ion source and discusses the impact of the magnetic field design on the source performance. The required equivalent proton beam current density of $40 \mathrm{~mA} / \mathrm{cm}^{2}$ was extracted at a moderate microwave power of $400 \mathrm{~W}$ with an optimized magnetic field.
\end{abstract}

Keywords: Neutron generator, Microwave ion source, Active interrogation

PACS: 29.25.Dz, 52.50.Sw, 52.80.Pi

\section{Introduction}

A high-yield, sealed-tube neutron generator (Fig. 1) was developed at the Lawrence Berkeley National Laboratory to address the needs of homeland security, non-proliferation, and nuclear safeguards applications. This generator was designed to produce high neutron yields, exceeding $5 \cdot 10^{11} \mathrm{n} / \mathrm{s}$ in D-T operation and reaching about $\sim 1 \cdot 10^{10} \mathrm{n} / \mathrm{s}$ in D-D operation [1] when operating at $100 \mathrm{kV}$ acceleration voltage and a $100 \mathrm{~mA}$ beam current impinging on a beam-loaded neutron production target. A V-shaped target geometry in combination with

*Presented on the 21st CAARI Conference. Fort Worth, Tx (USA) 2010 
beam extraction from the ion source through a large $60.6 \mathrm{~mm}^{2}$ slit serves to distribute the beam current and heat load over a larger area for better cooling of the target surface. Based on the assumption of a fully D-T loaded titanium target layer the amount of tritium in the generator is estimated to be roughly $200 \mathrm{Ci}$. However, this number depends on the thickness of the loaded target layer and could possibly be significantly lower for other targets.

Originally, this generator was equipped with an RF-driven plasma ion source that was specifically designed to operate at the low gas pressures required for sealed-tube generators. Initial testing of the generator was performed in D-D operation. While the RF-driven ion source was capable of providing the high beam current [1] the lifetime of the source was limited due to frequent sputtering. In recent years permanent-magnet microwave ion sources were developed [2]-[5] that offer significant advantages. They operate at low gas pressures of around $0.3 \mathrm{~Pa}$, produce high plasma densities and a high atomic species fraction at significantly lower power levels than RF-driven ion sources, and offer long lifetimes. This paper describes the development and design of a permanent-magnet microwave ion source for the high-yield neutron generator. The magnetic field design and its impact on the source performance is discussed, and initial results are presented.

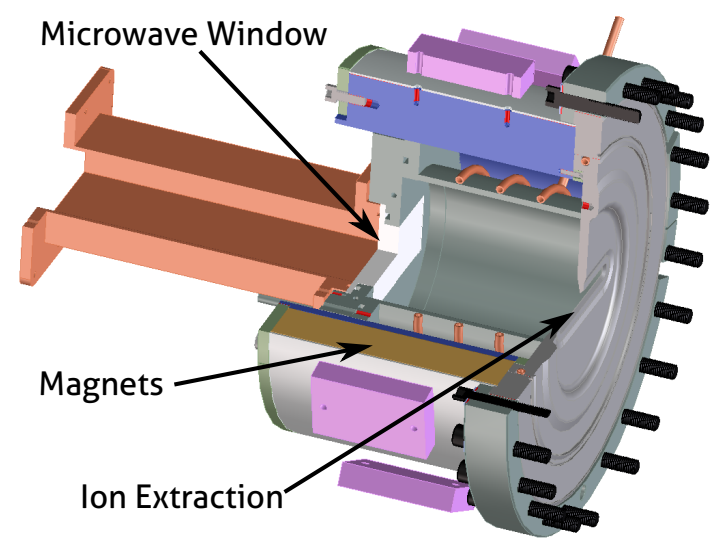

Figure 1: Microwave ion source. The plasma chamber is $100 \mathrm{~mm}$ long and 90 $\mathrm{mm}$ in diameter with a $60.6 \mathrm{~mm}^{2}$ ion extraction slit. It is surrounded by seven rectangular permanent magnet bars. A protective sheet can be inserted in front of the microwave window.

\section{Microwave Ion Sources}

Microwave-driven ion sources offer high extracted beam current densities, high atomic fractions, and high power efficiencies at low gas pressure [6]. Efficient 
ionization and high plasma densities are achieved when the electron-cyclotron resonance condition is met, i.e., at a magnetic field of $B=87.5 \mathrm{mT}$ for $2.45 \mathrm{GHz}$ microwave frequency operation. Permanent-magnet ion sources, without field coils and their power supplies, are compact and easy to operate, which makes them attractive for use in a neutron generator.

The ion source described here and schematically shown in Fig. 2, is based on an early proton microwave ion source that employed magnetic field coils [5]. In our design, the magnetic field is generated and shaped by permanent magnets and a ferromagnetic extraction electrode, such that the value of $87.5 \mathrm{mT}$ is reached very close to the extraction slit and at the vacuum window. Seven bars of permanent, rectangular NdFe-magnets surround the cylindrical plasma chamber (100 $\mathrm{mm}$ long and $90 \mathrm{~mm}$ in diameter, made of aluminum) to generate the magnetic field lines parallel to of the ion source axis. The plasma electrode extends radially beyond the magnets and matches the geometry of the existing neutron generator.

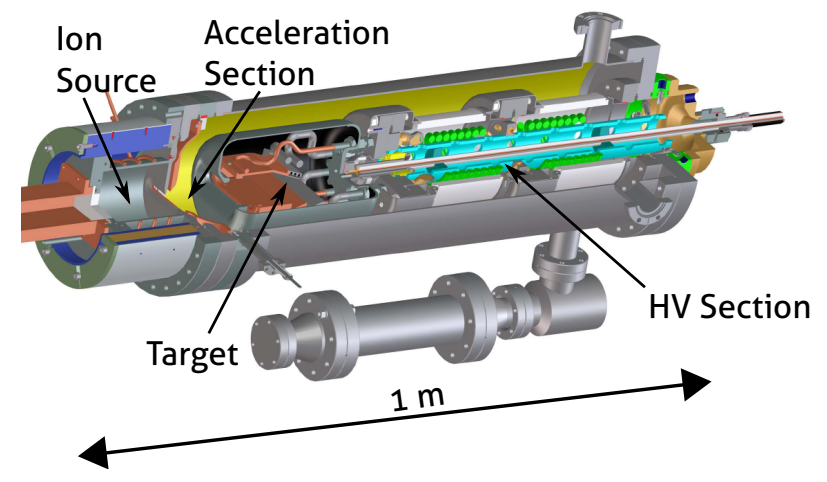

Figure 2: Neutron generator with microwave ion source.

This design avoids the need for extending the permanent magnets beyond the plasma electrode and allows us to simply attach the source with a conflat flange to the existing generator tube. The ferromagnetic plate also greatly lowers the magnetic field in the extraction and acceleration region thus minimizing its impact on secondary, backstreaming electrons and avoiding possible discharge problems. The magnetic design was done based on three-dimensional simulations with the code RADIA [7] that included the permanent magnets and all ferromagnetic materials. As can be seen in Fig. 3, the agreement between the simulated and the measured magnetic field along the axis of the source is very good. 


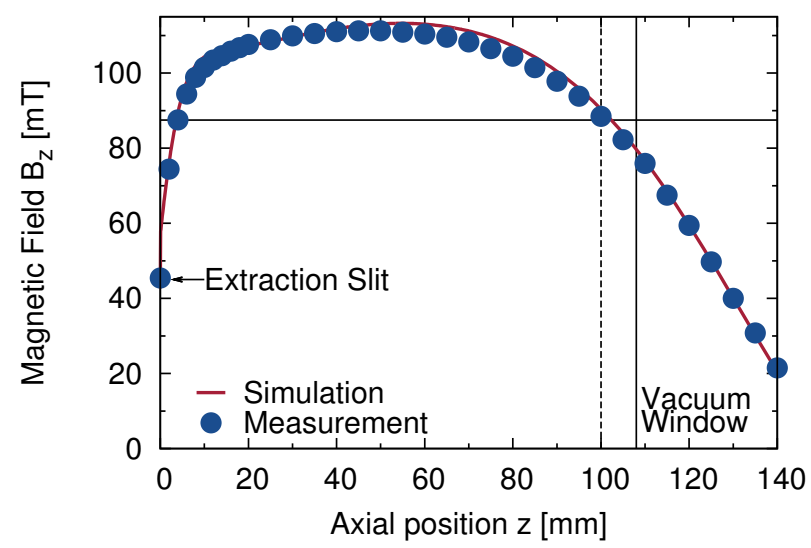

Figure 3: Magnetic Field. Measurement is shown in dots while the RADIA simulation is shown as a line. The dotted line marks the length of the chamber.

\section{Experimental Set-Up}

For testing and experimental characterization the microwave-driven ion source was mounted on a vacuum chamber equipped with an extraction system consisting of three electrodes and a Faraday cup for measuring the extracted beam current. Currents collected by the extraction electrodes were monitored to detect possible beam optics problems. Hydrogen gas was fed directly into the source chamber and pumped away through the vacuum chamber. Typical gas pressures inside the source were in the $0.1 \ldots 0.4 \mathrm{~Pa}$ range and in the extraction section about $5 \cdot 10^{-4} \mathrm{~Pa}$. The pressures were measured with a baratron cell and an ionization gauge, respectively.

The microwave generator provided up to $1.5 \mathrm{~kW}$ of continuous wave or pulsed microwave power that was guided through a circulator, a two-stub tuner, and a ridged waveguide [8] for optimum coupling of the microwave power into the source. Forward and reflected powers were monitored.

In the neutron generator the $100 \mathrm{~mA} \mathrm{D}-\mathrm{T}$ beam current is extracted through a $60.6 \mathrm{~mm}^{2}$ slit corresponding to an average beam current density of about $25 \frac{\mathrm{mA}}{\mathrm{cm}^{2}}$. However, in these experiments a proton beam was extracted through a $3 \mathrm{~mm}$ diameter aperture instead of the slit. 


\section{Results}

\subsection{Magnetic Field Variation}

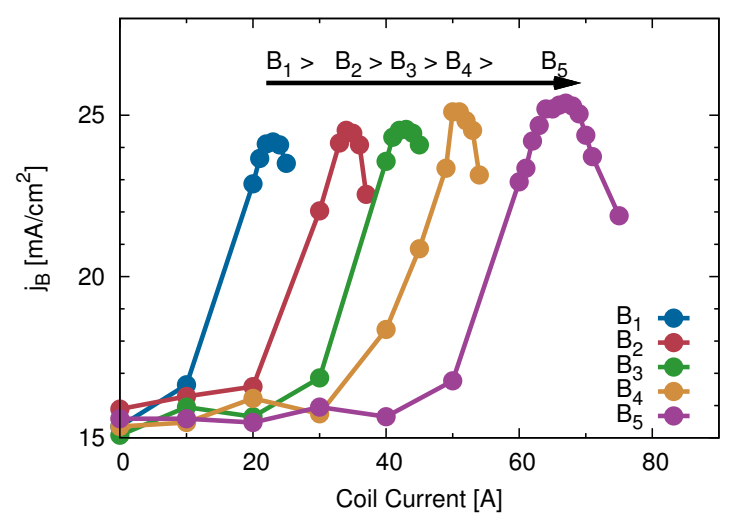

Figure 4: Extracted beam current densities for different magnetic fields. The permanent magnetic field is varied by different radial position of the steel plates while the field at the window is increased with the coil current.

In order to study the impact of magnetic field variations on source performance and to find the optimum magnetic configuration, we added a coil and ferromagnetic steel plates to the ion source. The coil was installed at the back of the source for changing the field predominantly at the microwave window and the steel plates were mounted outside of and parallel to the magnet bars as seen in Fig. 1 (pink blocks). By moving the plates radially in and out the magnetic field throughout the source volume is decreased or increased. Using the plates and the coil together the shape of the magnetic field as produced by the permanent magnets and the ferromagnetic steel extraction plate can be varied. Fig. 4 shows the change in extracted beam current with coil current for a set of different radial positions of the field weakening plates. In all cases the beam current was nearly doubled by increasing the coil current, i.e., the magnetic field at the window. The shift in optimal coil current for the different cases reflects the varying decreases in field strength due to the steel plates being at different radial positions. Fig. 5 shows simulations and measurements of magnetic fields along the axis of the source for. the coil currents that give the maximum beam current for each radial position of the field weakening plates. As seen in Fig. 5 there is a disagreement between simulation and measurement at distances more than $20 \mathrm{~mm}$ away from the microwave window. This was not further investigated, since this study focuses on the field in the vicinity of that window. The magnetic fields differ quite strongly throughout most of the ion source but for all cases the ECR condition is satisfied $\left(B_{z}=87.5 \mathrm{mT}\right)$ at the microwave window indicating that this is a crucial requirement for good source performance. We are now adjusting the permanent magnet configuration of our ion source to fine-tune the magnetic field at the window. 


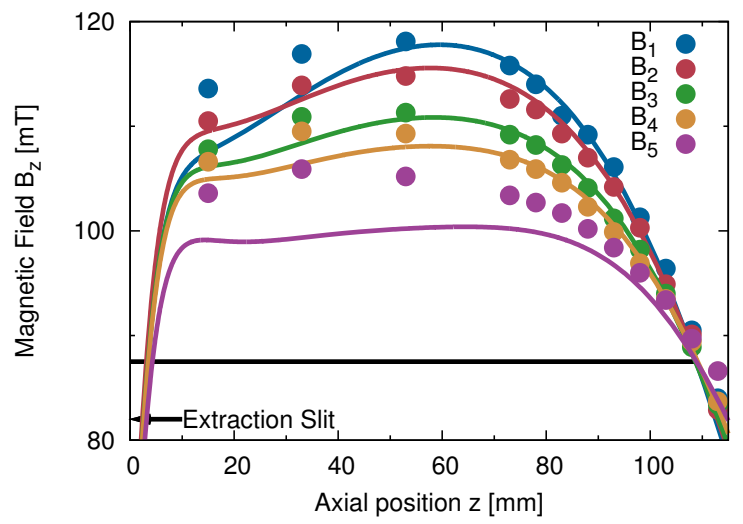

Figure 5: Magnetic fields for the peak current densities shown in Fig. 4. The measurements are shown as dots while the RADIA simulations are shown as lines. The ECR condition $(87.5 \mathrm{mT})$ and the position of the microwave window are marked in black.

\subsection{Variation of Microwave Power and Window Material}

The extracted beam current density was measured as a function of microwave power for two different window materials at a source pressure of $0.3 \mathrm{~Pa}$ shown in Fig. 6. For both materials, aluminum nitride (AlN) and quartz, the current density increases linearly with power. The proton current density corresponding to the neutron generator design value of a D-T current density of $25 \frac{\mathrm{mA}}{\mathrm{cm}^{2}}$ is 40 $\frac{\mathrm{mA}}{\mathrm{cm}^{2}}$. This value was reached at $400 \mathrm{~W}$ with an AlN window. When using a quartz window the required microwave power is about $30 \%$ higher.

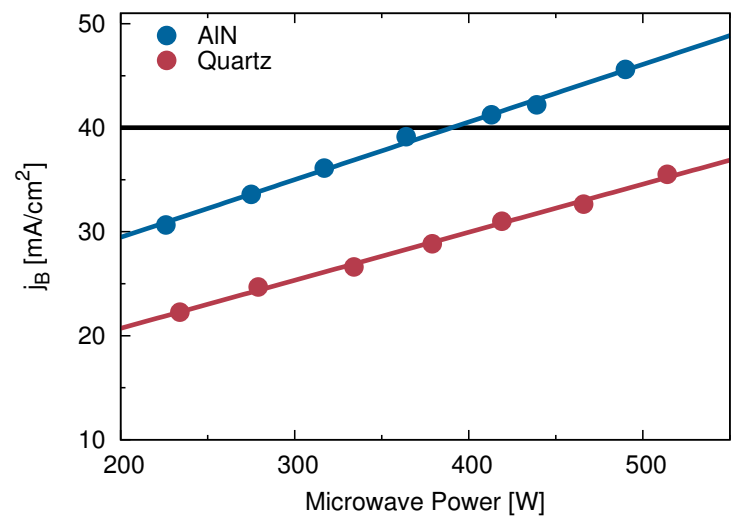

Figure 6: Change of extracted beam current with microwave power for an aluminum nitride and a quartz window. The target value of the beam current density is indicated by the black line. 


\section{Conclusion}

A permanent-magnet microwave ion source was developed for a compact highyield neutron generator. The required beam current density was extracted at a moderate microwave power of $400 \mathrm{~W}$ at the desired low gas pressures. This compares favorably with the power requirements $(\sim 1500 \mathrm{~W})$ of a previously developed RF-driven source $[9,1]$. It was further found that the use of an aluminumnitride vacuum window led to an approximately $30 \%$ higher extracted beam current than measured for a quartz window.

By varying the magnetic field we demonstrated that meeting the ECR condition directly at the microwave window is crucial for good source performance. This finding helped to optimize the permanent magnet configuration.

\section{Acknowledgments}

The authors would like to thank Qing Ji for many helpful discussions. This work was supported by the Office of Proliferation Detection (US DOE, NA-221) and the Director, Office of Science of the US Department of Energy at the Lawrence Berkeley National Laboratory under contract number DE-AC02-05CHI1231.

\section{References}

[1] B. Ludewigt et al., Nucl. Inst. and Methods in Phys. Res. B, 261, 830-834 (2007)

[2] W. Cornelius, Proc. of the IEEE Particle Accelerator Conf., 1884-188 (1999)

[3] Z. Song et al., Rev. Sci. Inst. 77, 03A305 (2006)

[4] Z. Yao et al., Rev. Sci. Inst. 79, 073304 (2008)

[5] O. Tuske et al., Rev. Sci. Inst. 79, 02B710 (2008)

[6] S. Jing et al., Rev. Sci. Inst. 72, 92-95 (2001)

[7] O. Chubar et al., J. Synchrotron Rad. 5, 481-484 (1998).

[8] T. Taylor and J. F. Mouris, Nucl. Inst. and Methods in Phys. Res. A, 336, 1-5 (1999)

[9] J. Reijonen et al., Appl. Rad. And Isotopes 63, 757-763 (2005) 